\title{
Management and Outcome of Craniofacial Fibrous Dysplasia in a Tertiary Neurosurgical Centre, Nigeria
}

\author{
Nasiru Jinjiri Ismail', Ali Lasseini, Aliyu Muhammad Koko and Bello B Shehu \\ Department of Neurosurgery, Regional Centre for Neurosurgery (RCN), Usmanu Danfodiyo University \\ Teaching Hospital Sokoto, Nigeria
}

*Corresponding author: Dr. Nasiru Jinjiri Ismail, Department of Neurosurgery, Regional centre for Neurosurgery, Usmanu Danfodiyo University Teaching Hospital Sokoto, Nigeria

\begin{abstract}
Background: Fibrous dysplasia is an aberration in normal bone development that results from a defect in osteoblastic differentiation and maturation originating in a mesenchymal precursor; it's characterized by foci of abnormal fibro-osseous proliferation that can affect any area of the calvaria. Craniofacial fibrous dysplasia is a rare condition that can presents with craniofacial swelling and disfigurement. Usually, cranial computerised tomography scan is needed for radiological evaluation and excision and cranioplasty offers acceptable outcomes.
\end{abstract}

Objective of study: The objective of the study was to describe the clinical and radiological characteristics and outcome of management of craniofacial fibrous dysplasia in our centre.

Materials and methods: Medical records of patients with craniofacial fibrous dysplasia managed in our centre between January 2007 to December 2016, were reviewed retrospectively. Biodemographics of the patient, imaging and histological findings and surgical outcomes were noted and analysed.

Results: We found eleven cases of histologically confirmed craniofacial fibrous dysplasia over the study period. Seven cases were females with male-to-female ratio of 1:1.8. The mean age of presentation was 20 years \pm 2 S.D. The commonest reason for presentation was cranio-facial disfigurement. All cases were polyostotic involving fronto-orbital area. Total excision and cranioplasty using polymethyl methacrylate was done for all patients. The most common complication was postoperative seroma, treated with antibiotic, aspiration and pressure dressing. There were two cases $(18 \%)$ of implant infection that warranted removal of infected implants and subsequent cranioplasty three month later.

Conclusion: The study shows that cranio-facial fibrous dysplasia was commonly seen in first 3 decades of life and affects females much more than the male counterpart. Total excision and cranioplasty using polymethyl methacrylate confers acceptable results.

\section{Keywords}

Fibrous dysplasia, Craniofacial, Management outcome

\section{Introduction}

Fibrous dysplasia is a benign fibrous-osseous disease in which the normal bone matrix is replaced by fibroblastic proliferation [1-3]. The incidence rates of fibrous dysplasia are reported at 1:4000 to $1: 10,000$ populations. Fibrous dysplasia accounts for $2.5-10 \%$ of all bone tumours $[1,4]$. The disease was described as "osteitis fibrosa generalisata" by von Recklinghausen in 1891 and subsequently coined as "fibrous dysplasia" by Lichtenstein in $1938[1,4]$.

The pathogenetic mechanism involves an arrest of differentiation of immature bone marrow cells due to somatic missense mutation in the gene GNAS1 on chromosome 20q13.2-13.3; the mutation at position 201 of Gs $\alpha$ results in substitution of cystein or histidine by arginine [5-7].

Fibrous dysplasia can involve single bone (monostotic form), multiple bones (polyostotic form) or presents in syndromic form, such as Mccune Albright syndrome (where there is associated café au lait spot and endocrinopathies), Mazabraud syndrome (fibrous dysplasia with intramuscular myxomas) $[5,8,9]$. Majority of cases of fibrous dysplasia are seen in the first three decades of life, with slight female preponderance [4]. The most

Citation: Ismail NJ, Lasseini A, Koko AM, Shehu BB (2020) Management and Outcome of Craniofacial Fibrous Dysplasia in a Tertiary Neurosurgical Centre, Nigeria. Neurosurg Cases Rev 3:034. doi. org/10.23937/2643-4474/1710034

Accepted: May 14, 2020; Published: May 16, 2020

Copyright: (c) 2020 Ismail NJ, et al. This is an open-access article distributed under the terms of the Creative Commons Attribution License, which permits unrestricted use, distribution, and reproduction in any medium, provided the original author and source are credited. 
common presenting clinical features are craniofacial swelling and disfigurement; visual impairment due to optic nerve compression remains the most devastating consequences of craniofacial fibrous dysplasia $[1,4,6]$.

Computerised tomography (CT) scan is an invaluable imaging modality for the assessment of craniofacial fibrous dysplasia because of its ability to delineate bony structures accurately $[1,9]$. The characteristic CT scan appearance of fibrous dysplasia consists of: ground glass pattern (56\%), homogenously dense pattern (23\%) and cystic appearance (21\%) [1]. Magnetic resonance imaging provides more detailed soft tissue resolutions than CT scan but, it is not recommended because low signal from bone affected by fibrous dysplasia could be misdiagnosed for neoplasms [1,9].

Treatments of fibrous dysplasia can be non-surgical or surgical. Non-surgical treatments include observation and regular monitoring with $\mathrm{CT}$ scan, use of bisphosphonates like pamidronate, and use of analgesics (non-steroidal anti-inflammatory drugs) for control of pain. The non-surgical treatment options are employed in patients that have quiescent (stable, no growth) disease or those who refuse surgery [2]. Surgical treatment depends on the following factors: location of the growth, cosmetic disturbance, functional disruption and patient's preference, general condition of the patient, available facilities and the experience of the surgeon [1]. The craniofacial skeleton has been classified into four zones based on the anatomical area involved [1]. Zone 1 includes fronto-orbital, zygomatic and upper maxillary regions; zone 2 involves the hair-bearing cranium; zone 3 represents the central cranial base; and zone 4 is teeth-bearing regions of the maxillary alveolus and mandible. Total excision of the affected bone is recommended for zone 1 and conservative or shaving is advocated for the remaining zones [1]. Generally, the attending surgeon must choose the surgical option that will provide the patient the desired outcome as well as preserve the functions of adjacent structures.

\section{Problem statement}

Craniofacial fibrous dysplasia is one of the neurosurgical conditions seen in our environment. There is been paucity of data about the clinical profile and outcomes of craniofacial fibrous dysplasia in our subregion thereby making development of treatment guidelines difficult.

\section{Rationale of the study}

The result of this study will bring out the data about craniofacial fibrous dysplasia in our settings which will serves as baseline for management of patients and subsequent research in this field.

\section{Objective of study}

The objective of the study was to describe the clinical and radiological characteristics and outcome of man- agement of craniofacial fibrous dysplasia in our centre.

\section{Materials and Methods}

This is retrospective descriptive study of patients with craniofacial fibrous dysplasia managed between January 2007 to December 2016, in a regional centre for neurosurgery, Usmanu Danfodiyo University Teaching Hospital Sokoto, Nigeria. Records of patients with craniofacial fibrous dysplasia were included in a proforma while cases of recurrent fibrous dysplasia and fibrous dysplasia outside craniofacial region were excluded. Variables such as biodemographic characteristics, clinical features, radiological and histopathological findings, and outcomes of treatment (postoperative seroma, implant infection and cosmesis) were analysed by simple descriptive analysis using SPSS IBM version 20.0 for windows. The surgical access was gained a bicoronal incision for the patients, lesion exposed and excised completely. No dural breach recorded and sinus was cranialised and plugged with pedicled pericranial flap and cranioplasty done using polymethyl methacrylates. Post-operatively patients were given antibiotics, analgesics and wound care. Passive, closed drain was inserted intraoperatively and removed after forty-eight hours. Most patients were followed up for up to two years but some were lost to follow up as early as 6 months post-surgery.

\section{Results}

Eleven patients with craniofacial fibrous dysplasia were managed during the study period. These consisted of seven females and four males giving a male-tofemale ratio of $1: 1.8$. The age range was 6 to 40 years, with mean age of 20 years \pm 2 S.D. The universal presenting clinical features were craniofacial swelling and disfigurement and diplopia (Figure 1), in addition, two

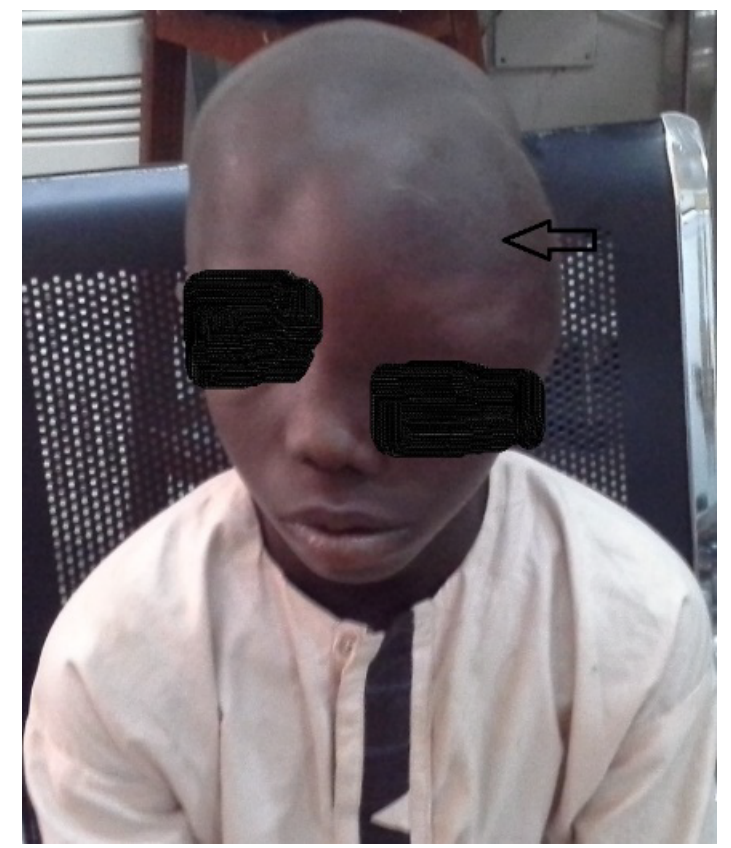

Figure 1: Clinical photograph a patient with left fronto-orbital mass. 


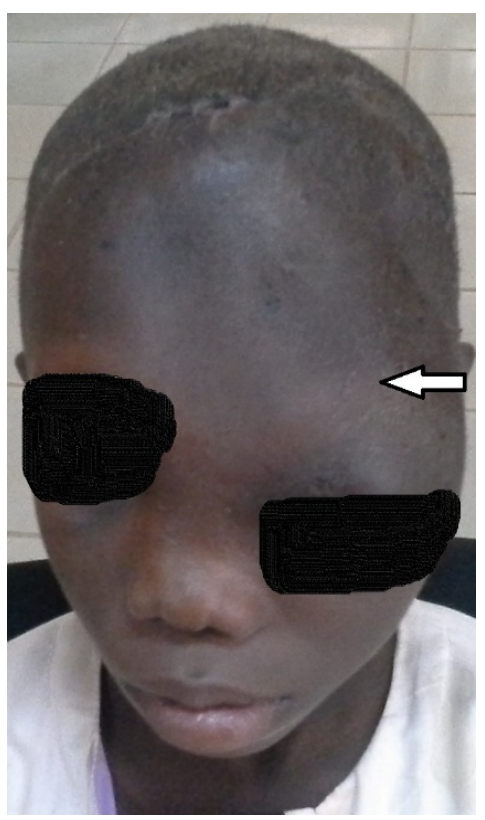

Figure 2: Post-operative clinical photograph (10 days after surgery) showing residual seroma.

patients complained of headache. No focal neurological deficits observed. The size of the lesion was more than $20 \mathrm{~cm}$ (widest diameter) in majority of patients $(9 / 11)$, less than $20 \mathrm{~cm}$ in its widest diameter in two patients. The lesion was located on the left side in seven patients and on the right in the remaining cases. Cranial computerised tomography scan was used for diagnosis and assessment of extent of the lesions for all patients. The CT scan findings were mixed density bony lesion with expansion of the diploe and adjacent sinus. All cases involved the fronto-orbital region, had mixed consistency of hard and soft area and poorly demarcated intraoperatively. A total excision and cranioplasty using polymethyl methacrylate was done. The most common postoperative complication was seroma in nine patients (9/11) (Figure 2), which was treated by aspiration, antibiotic therapy and turban pressure dressing. Other postoperative complication was superficial surgical infection (3/11) that was treated with antibiotics and wound dressing. There were two (18\%) cases of implant infection that warranted removal of the infected methyl methacrylate implant and subsequent cranioplasty three months later. Acceptable cosmesis was recorded in nine patients. The duration of hospital stay was 2-3 weeks in 8 patients, less than 2 weeks in 2 patients and more than 3 weeks in one patient. The long-term postoperative outcome observed was implant displacement in one patient $(1 / 11)$ seen after 8 years of surgery.

\section{Discussion}

In this study there was a slight female preponderance (male-to-female ratio $1: 1.8$ ), which is consisted with reports from other comparable studies $[4,6]$. When all age groups were considered, there was marked preference of craniofacial fibrous dysplasia for the first three decades of life (Figure 1) $[4,8]$. This was the case in our

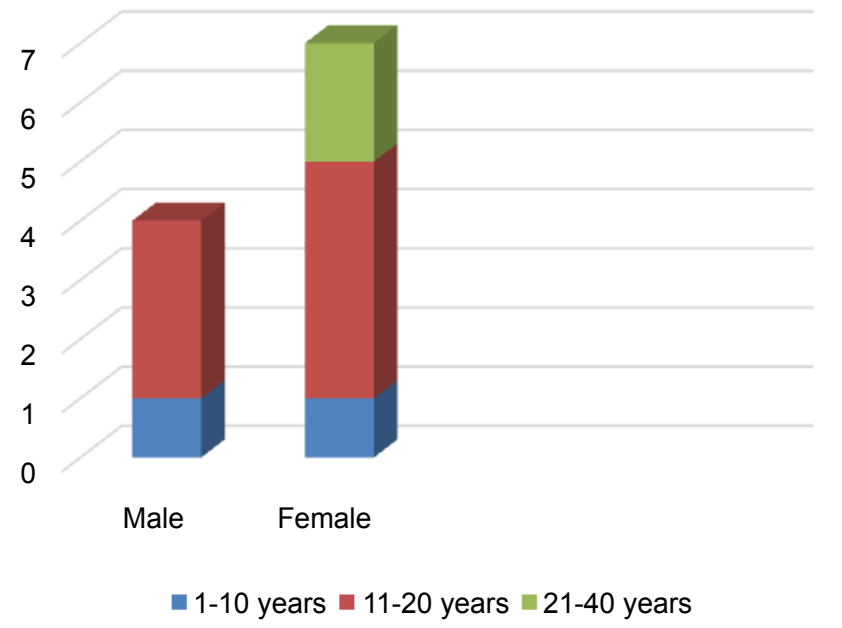

Figure 3: Age/Sex distribution of cases.

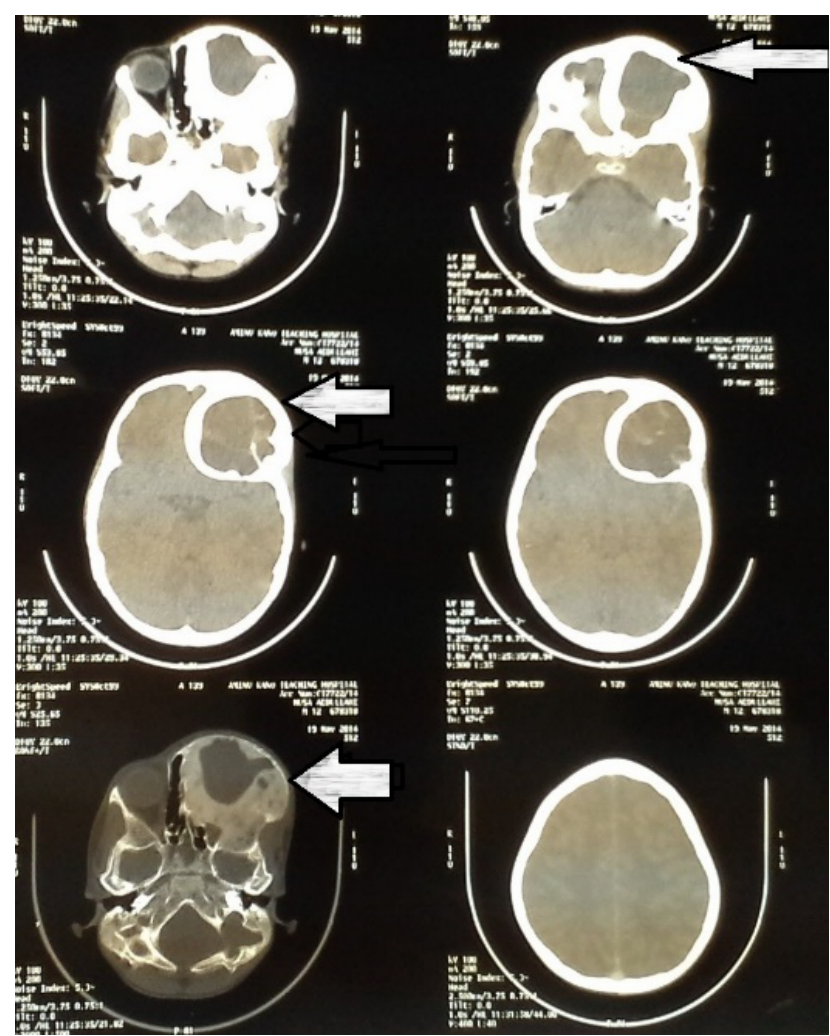

Figure 4A: Axial cut CT scan showing left fronto-orbital fibrous dysplasia.

study where $90 \%$ of craniofacial fibrous dysplasia were seen in patients aged 30 and below (Figure 3).

The primary reasons for seeking treatment in all patients were craniofacial swelling and aesthetic disturbance [10]. Though, reports from previous studies were in tandem with our findings other remarkable clinical features were visual impairment, pain and pathological fractures which were absent in all our cases $[1,4,11]$. Monostotic fibrous dysplasia has been reported to occur four times more often that polyostotic type however, polyostotic fibrous dysplasia affects craniofacial regions in $90 \%$ of the cases $[2,6,12]$. This conforms to the findings of our study of polyostotic fibrous dysplasia in all the cases (Figure 4A and Figure 4B). 


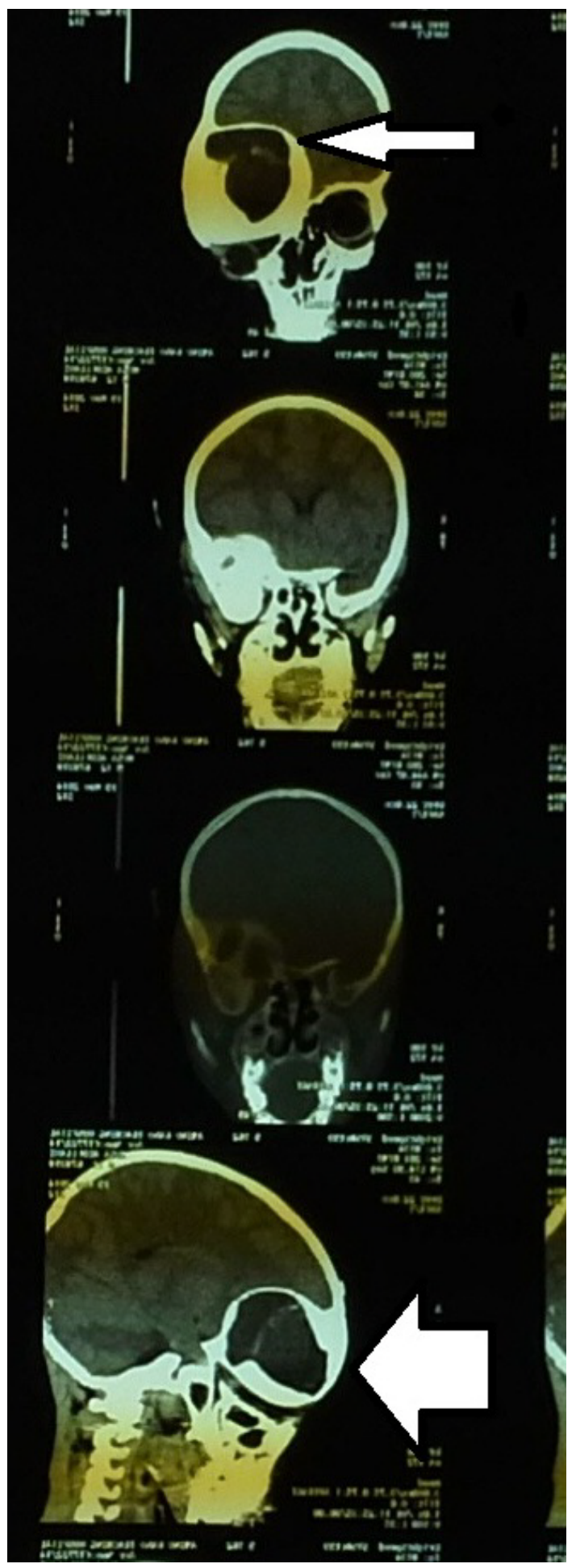

Figure 4B: Coronal and sagittal views CT scan of a patient with fronto-orbital fibrous dysplasia.

A total excision and cranioplasty using polymethyl methacrylate were done for all patients (Figure 5). Methyl methacrylate implants was used because it is affordable, available and easy to customise to the desired shape. Two cases of implant infection occurred within three months of surgery; the infected implants were removed and re-operated (cranioplasty using methyl methacrylate) 3 months later to allow for control of infection and healing of inflamed tissues. Satisfactory outcome measured by acceptable cosmesis and absence of implant infection within 3 months of surgery was recorded in the majority of patients. The present series

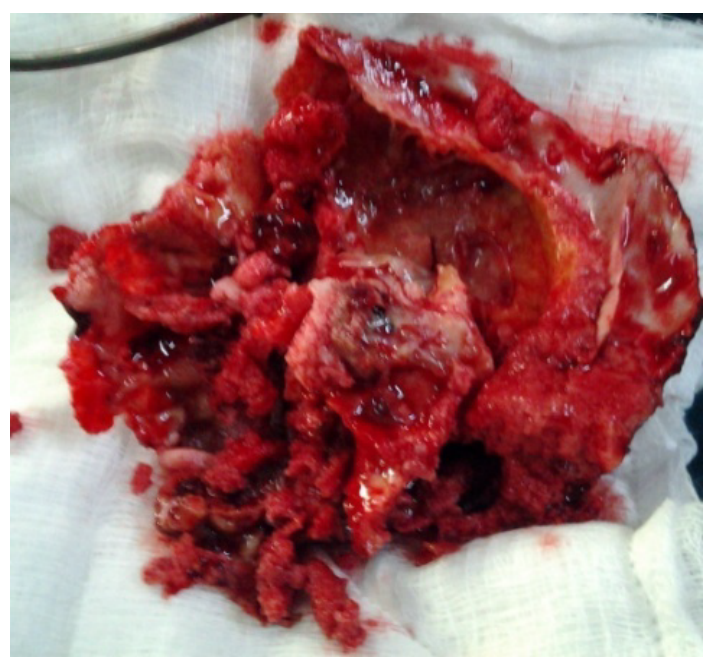

Figure 5: Clinical photograph showing gross fibrous dysplasia specimen after excision.

described the clinical and radiological features as well as outcomes of management craniofacial fibrous dysplasia in our setting. This would serve as foundation for future research in this neurosurgical condition and has contributed a data in literature on craniofacial fibrous dysplasia globally while given peculiarities of our environment.

\section{Conclusion}

Surgical treatment of craniofacial fibrous dysplasia is challenging to both the surgeons and patients, this is due to aesthetic disfigurement to the patient and the surgeon's task of reconstruction of the disfigured anatomy to meet patient's desire. The disease has preponderance for females and young individuals in first 3 decades of life. Patients usually present due to cosmetic embarrassment and swelling. Total excision and cranioplasty using polymethyl methacrylate offers acceptable cosmesis.

\section{Conflict of Interest}

None.

\section{Acknowledgement}

We thank all staff of neurosurgery department for their immense contribution in care of our patients.

\section{References}

1. Yu-Ray Chen, Cheng-Nen Chang, Ying-Chien Tan (2006) Craniofacial fibrous dysplasia: An update. Chang Gung Med J 29: 543-549.

2. JS Lee, EJ Fitz Gibbon, YR Chen, HJ Kim, LR Lustig, et al. (2012) Clinical guidelines for the management of craniofacial fibrous dysplasia. Orphanet J Rare Dis 7: S2.

3. Aakarsh Jhamb, Sujata Mohanty, Parul A Jhamb (2012) Craniofacial fibrous dysplasia. J Oral Maxillofacial Pathol 16: 465-469.

4. Suresh Menon, Srihari Venkatswamy, Veena Ramu, Khurshida Banu, Sham Ehtaih, et al. (2013) Craniofacial fibrous dysplasia: Surgery and literature review. Ann Maxillofac Surg 3: 66-71. 
5. Moran Amit, Michael T Collins, Edmund J FitzGibbon, John A Butman, Dan M Fliss, et al. (2011) Surgery versus watchful waiting in patient with craniofacial fibrous dysplasia - a meta-analysis. PLoS One 6: e25179.

6. Janice S Lee, Edmond Fitzgibbon, John A Butman, Craig R Dufresne, Harvey Kushner, et al. (2002) Normal vision despite narrowing of the optical canal in fibrous dysplasia. N Engl J Med 347: 1670-1676.

7. Promphan Pruksakorn, Shanop Shuangshoti, Rungsak Siwanuwatn, Sukalaya Lerdlum, Sarat Sunthornyothin, et al. (2010) Craniofacial fibrous dysplasia. Intern Med 49: 249250.

8. Jonathan Mangion, Sarah Edkins, Alastair N Goss, Michael R Stratton, Adrienne M Flanagan (2000) Familial craniofa- cial fibrous dysplasia: Absence of linkage to GNAS1 and the gene for cherubism. J Med Genet 37: E37.

9. BD Daly, CC Chow, CS Cockram (1994) Unusual manifestation of craniofacial fibrous dysplasia: Clinical, endocrinological and CT features. Postgrad Med J 70: 10-16.

10. Mark S Greenberg (2010) Handbook of neurosurgery. ( $7^{\text {th }}$ edn), Thieme medical publishers, New York.

11. Morrissey DD, Talbot JM, Schlevning AJ (1997) Fibrous dysplasia of the temporal bone: Reversal of sensorineural hearing loss after decompression of internal auditory canal. Laryngoscope 107: 1336-1340.

12. Sadeghi SM, Husseini SN (2011) Spontaneous conversion of fibrous dysplasia into osteosarcoma. J Craniofac Surg 22: 959-961. 